\title{
Orthotopic Kidney Auto-Transplantation in a Porcine Model Using 24 Hours Organ Preservation And Continuous Telemetry
}

\author{
Wen-Jia Liu ${ }^{*}, 1,2$, Lisa Ernst ${ }^{\star}{ }^{2}$, Benedict Doorschodt ${ }^{2}$, Jan Bednarsch ${ }^{1}$, Felix Becker ${ }^{3}$, Richi Nakatake ${ }^{2}$, Yuki Masano $^{2}$, Ulf \\ Peter Neumann ${ }^{1,4}$, Sven Arke Lang ${ }^{1}$, Peter Boor ${ }^{5}$, Isabella Lurje ${ }^{6}$, Georg Lurje ${ }^{1,6}$, René Tolba ${ }^{2}$, Zoltan Czigany ${ }^{1,2}$ \\ ${ }^{1}$ Department of Surgery and Transplantation, Faculty of Medicine, University Hospital RWTH Aachen ${ }^{2}$ Institute for Laboratory Animal Science, Faculty of \\ Medicine, University Hospital RWTH Aachen ${ }^{3}$ Department of General-, Visceral-, and Transplantation Surgery, University Hospital Muenster ${ }^{4}$ Department \\ of Surgery, Maastricht University Medical Centre (MUMC) ${ }^{5}$ Institute of Pathology, Faculty of Medicine, University Hospital RWTH Aachen ${ }^{6}$ Department of \\ Surgery, Campus Charité Mitte | Campus Virchow-Klinikum, Charité-Universitätsmedizin \\ *These authors contributed equally
}

\section{Corresponding Author}

Zoltan Czigany

zczigany@ukaachen.de

\section{Citation}

Liu, W.J., Ernst, L., Doorschodt, B., Bednarsch, J., Becker, F., Nakatake, R., Masano, Y., Neumann, U.P., Lang, S.A., Boor, P., Lurje, I., Lurje, G., Tolba, R., Czigany, Z. Orthotopic Kidney AutoTransplantation in a Porcine Model Using 24 Hours Organ Preservation And Continuous Telemetry. J. Vis. Exp. (162), e61591, doi:10.3791/61591 (2020).

\section{Date Published}

August 21, 2020

DOI

$10.3791 / 61591$

URL

jove.com/video/61591

\section{Abstract}

In the present era of organ transplantation with critical organ shortage, various strategies are employed to expand the pool of available allografts for kidney transplantation (KT). Even though, the use of allografts from extended criteria donors (ECD) could partially ease the shortage of organ donors, ECD organs carry a potentially higher risk for inferior outcomes and postoperative complications. Dynamic organ preservation techniques, modulation of ischemia-reperfusion and preservation injury, and allograft therapies are in the spotlight of scientific interest in an effort to improve allograft utilization and patient outcomes in KT.

Preclinical animal experiments are playing an essential role in translational research, especially in the medical device and drug development. The major advantage of the porcine orthotopic auto-transplantation model over ex vivo or small animal studies lies within the surgical-anatomical and physiological similarities to the clinical setting. This allows the investigation of new therapeutic methods and techniques and ensures a facilitated clinical translation of the findings. This protocol provides a comprehensive and problem-oriented description of the porcine orthotopic kidney auto-transplantation model, using a preservation time of 24 hours and telemetry monitoring. The combination of sophisticated surgical techniques with highly standardized and stateof-the-art methods of anesthesia, animal housing, perioperative follow up, and monitoring ensure the reproducibility and success of this model. 
Since the first successful human renal transplantation between identical twins in 1954, performed by the pioneering group of the Nobel prize laureate surgeon Joseph Murray ${ }^{1}$, kidney transplantation (KT) has evolved as the mainstay of treatment for patients with end-stage renal disease (ESRD) ${ }^{2}$. KT shows superior long-term clinical outcomes and quality of life compared to dialysis ${ }^{2}$. Short- and long-term survival rates after KT improved continuously, due to advances in surgical techniques, organ preservation, immunosuppressive therapy, and critical care, hence KT became widely available on a global scale $2,3,4$.

Due to critical organ shortage, there is a continuously increasing gap between allograft supply and demand ${ }^{3,5,6}$. In 2018, approximately 12,031 patients were waiting for KT in Germany, however, only less than $20 \%$ (2,291 patients) could receive a donor kidney due to the extreme shortage in organs for transplantation ${ }^{7}$. Unfortunately, not only the absolute number of organ donors, but also the general quality of the allografts offered for transplantation have declined in the past decades 8,9 . An increasing tendency was observed in the numbers of predamaged or "marginal" kidney allografts that had to be accepted for transplantation ${ }^{10}$. The use of ECD allografts may reduce waiting time and waiting list morbidity and mortality, it is, however, associated with an increased incidence of graft-related complications such as primary graft non-function (PNF) and/or delayed graft function $(\mathrm{DGF})^{8,9,10}$. Further research is essential to optimize allograft utilization, expand the donor pool and protect and recondition marginal allografts which ultimately may improve patient outcomes ${ }^{3,6}$.

Due to the resource-intensive and complex nature of large animal transplantation models, a large number of studies are performed using small animals or in ex vivo settings $11,12,13,14,15$. Although these models can deliver important scientific data, the translation of these findings to the clinical setting is often limited. The porcine model of orthotopic kidney auto-transplantation is a well-established and reproducible model that allows to test new innovative treatment approaches in a clinically relevant in vivo setting, with potentially longer follow-up periods and abundant possibilities for repetitive sample collection ${ }^{16}, 17$. Beyond the advantage of the comparable size, which allows relatively direct translation into the clinical setting (particularly for medical device development and drug dosage), the surgicalanatomical and physiological similarities in terms of ischemiareperfusion injury (IRI) response and kidney damage, support the use of this model in translational research ${ }^{17,18,19}$. This model also provides an excellent training opportunity to prepare young transplant surgeons for the technical challenges of clinical organ transplantation ${ }^{20}$.

There are also multiple differences compared to the human setting and various technical modifications of the model can be found in the literature $16,17,19,20,21$. This article comprehensively describes technical details, pitfalls, and recommendations which can aid to establish the model of porcine orthotopic kidney auto-transplantation. The described telemetry and video monitoring method as well as our specifically designed housing facility allows a close-up severity assessment and clinical observation of the animals. The use of a percutaneous urinary catheter and designated porcine jackets provide the possibility of a detailed assessment of kidney function without the use of metabolic cages. These technical modifications are described as potential solutions to comply with the modern challenges of the $3 \mathrm{R}$ principle (Replacement, Reduction and 
Refinement) and improve animal experiments using large animal models ${ }^{22}$.

\section{Protocol}

The present study was designed according to the principles of the ARRIVE (Animal Research: Reporting of In Vivo Experiments) guidelines ${ }^{23}$. Experiments were performed in accordance with the institutional guidelines and the German federal law regarding the protection of animals. The full ethical proposal was approved by the responsible authorities (Governmental Animal Care and Use Committee, LANUV NRW - "Landesamt für Natur, Umwelt und Verbraucherschutz Nordrhein-Westfalen", Recklinghausen, Germany, Protocol ID: 81-02.04.2018.A051). All animals in the present study received humane care according to the principles of the "Guide for the Care and Use of Laboratory Animals" (8th edition, NIH Publication, 2011, USA) and the Directive $2010 / 63 / E U$ on the protection of animals used for scientific purposes (Official Journal of the European Union, 2010). Female German landrace pigs were obtained from a hygienically optimized barrier breeding facility (Heinrichs $\mathrm{GbR}$, Heinsberg, Nordrhein-Westfalen). Figure 1 depicts the summary of the described experimental protocol.

\section{Animals and housing}

1. Use female German landrace pigs (or comparable) for this protocol. Deliver the animals to the research facility 14 days before the first surgery (telemetry implantation) for acclimatization and house them in a temperature- and humidity-controlled barrier environment with a $12 \mathrm{~h}$ light and dark cycle ( Figure 2).

2. Mount two telemetry receivers on the ceiling of the room which allows the registered data to be transferred directly to a PC located in the observation room. Ensure that animals are observed visually during the regular visits by the veterinary officers and by the animal caretaker in charge (every $8 \mathrm{~h}$ and on-demand).

NOTE: Furthermore, in this experiment a realtime camera footage with integrated thermal imaging connected to the local network was used. Details of the housing facility used in this study are depicted in Figure 2.

\section{Basic techniques and common procedures}

1. Fast the animals overnight before surgery.

2. Premedicate by an initial intramuscular injection of azaperone $(4 \mathrm{mg} / \mathrm{kg})$ and atropine $(0.1 \mathrm{mg} / \mathrm{kg})$, followed by an injection of ketamine $(15 \mathrm{mg} / \mathrm{kg}) 10 \mathrm{~min}$ later.

3. After premedication, weigh the animal and transfer it directly from the housing facility to the central OR facility anesthesia preparation room.

4. Cannulate one of the large ear veins using an $18 \mathrm{G}$ peripheral venous catheter. Monitor the animal by a standard ECG and pulse oxymetry.

5. Initiate the anesthesia with propofol $(3 \mathrm{mg} / \mathrm{kg})$.

6. Expose the vocal cord with a laryngoscope and insert a $7.5 \mathrm{~mm}$ endotracheal tube. The cuff is blocked with air according to manufacturer's recommendations.

7. Insert an oro-gastric drainage tube to remove fluid and air from the stomach.

8. Insert a urinary catheter via the urethra.

9. Subsequently, trim the skin in the area of the surgical incision.

10. Apply eye ointment to prevent drying of the cornea during surgery. 
11. After orotracheal intubation, maintain anesthesia with isoflurane (final expiratory 1.45-2.0 Vol.\%) and fentanyl (3 $-7.5 \mu \mathrm{g} / \mathrm{kg} / \mathrm{h})$.

12. Ensure active intraoperative temperature control of the animal by a heating pad and using warmed air. Insert a rectal probe to monitor body temperature (target temperature $36.5^{\circ} \mathrm{C}-37.5^{\circ} \mathrm{C}$ ).

13. Administer antibiotic prophylaxis using cefuroxime (35 $\mathrm{mg} / \mathrm{kg}$ i.v.). Infuse Ringer solution at $4 \mathrm{~mL} / \mathrm{kg} / \mathrm{h}$ and increase to $8 \mathrm{~mL} / \mathrm{kg} / \mathrm{h}$ after skin incision. Administer a prophylactic dose of pantoprazole $(40 \mathrm{mg}$ i.v.) over the ear vein access.

14. Perform all surgical procedures under sterile conditions according to the general principles of surgical asepsis and antisepsis. Disinfect the surgical field with povidoneiodine solution and cover with surgical drapes.

\section{Telemetry implantation}

1. Prepare the animal for surgery following the steps described under section 2 of the protocol and confirm proper anesthesia by a decreasing heart rate and a lack of conscious movement of the animal.

2. Collect blood and urine samples to determine individual baseline lab values.

3. Mark the incision sites using a permanent marker.

4. To implant the arterial sensor of the telemetry transponder, perform a $3-4 \mathrm{~cm}$ incision in the groin. Expose and dissect the artery in a $360^{\circ}$ fashion.

5. Using an Overholt clamp pull through two-vessel loops below the artery and secure them with mosquito clamps.

6. After making an arteriotomy using \#11 blade scalpel, insert the arterial sensor. Close the arteriotomy using 5-0 polypropylene suture with single knot sutures and secure the arterial sensor using one of these sutures.

7. Make a 3-4 cm large incision on the left flank of the animal and create a subcutaneous pouch for the transponder by blunt dissection.

8. Tunnel the telemetry transponder to the flank and fix it to the muscle fascia (3-0 polypropylene, single knot). Tunnel the red and white ECG electrodes to the right and left side of the thorax. Make two $1 \mathrm{~cm}$ incisions and secure the electrodes in the muscle tissue to ensure a good ECG signal with single knot sutures (3-0 polyglactin).

9. Commence registration of telemetry data and check the various signals (e.g., body temperature registered by the transponder body itself, arterial blood pressure, and ECG signals).

10. Close the incisions in the groin, on the left flank and the two small thoracal incisions using muscle and subcutaneous sutures (3-0 polyglactin) and close the skin using a non-absorbable monofilament suture (e.g., 2-0 Prolene).

11. Use a spray film dressing to seal the incision sites.

12. At this time point prepare the animal to wear a designated porcine jacket which the animal wears for the rest of the study period. Replace jackets with a clean jacket following every surgical intervention.

NOTE: To record stable baseline data, telemetry transponders are implanted 14 days before the index surgery (left nephrectomy, please also see Discussion).

\section{Nephrectomy and kidney graft retrieval}

1. Prepare the animal for graft retrieval surgery following the procedures described in section 2. 
2. After the induction of anesthesia, cannulate the external jugular vein. Following the sterile disinfection of the surgical field, a $4 \mathrm{~cm}$ incision is made on the right side in the jugular groove.

3. Dissect subcutaneous tissue and muscle to expose the external jugular vein.

4. Expose and dissect the vein in a $360^{\circ}$ fashion.

5. Using an Overholt clamp pull through two-vessel loops below the vein and secure them with mosquito clamps.

6. Tunnel the jugular catheter to the back of the animal. For this, position the pig on its left side.Use the Seldinger method to insert the jugular catheter.

7. Close the opening on the vein and secure the catheter using 5-0 polypropylene suture.

8. Close the incision in two layers (e.g., 3-0 polyglactin for the muscle and subcutaneous and 2-0 polypropylene for the skin).

9. Secure the catheter to the skin with multiple sutures $(2-0$ polypropylene).

10. Test the jugular vein catheter for free aspiration and injection. Subsequently, switch the intravenous line from the ear vein cannula to the central venous line.

11. Following surgical disinfection and draping, perform a median laparotomy to open the abdomen $(25-30 \mathrm{~cm})$. Use a standard abdominal retractor to expose the surgical field.

12. Use wet and warm abdominal towels to cover the colon and the small bowel. Ask the second assistant to hold the bowel to the direction of right hemi-abdomen exposing the kidney and its vascular structures.
13. Open the peritoneal layer and dissect the left kidney and the ureter from any adherent tissue using the monopolar cautery, bipolar forceps, and fine scissors.

14. Ligate and divide the left ureter (3-0 polyglactin) leaving an at least 10 to $12 \mathrm{~cm}$ long segment.

15. Complete the dissection of the left renal vein(s) and artery to their origin from inferior vena cava and aorta, respectively.

NOTE: Avoid injury and opening of the large lymphatic vessels in this anatomical region. Also be aware of a potential injury to the azygo-lumbar vein joining to the renal vein near to its origin from the vena cava.

16. Dissect and ligate the azygo-lumbar vein between two ligatures (3-0 polyglactin).

17. Prepare for the back-table dissection using a bowl of ice and a sterile cover.

18. To retrieve the graft kidney, clamp the renal artery and the renal vein close to the aorta and the vena cava with vascular clamps. Remove the kidney graft by cutting the vessels with a scissor close to the clamps then hand the kidney over to the back-table team.

19. Close the stump of the renal artery using a 5-0 polypropylene suture. Close the renal vein using a twolayer continuous suture with 5-0 polypropylene. Remove the vascular clamps.

20. After checking the area for bleeding or lymphatic leakage, close the abdomen in 4 layers.

NOTE: Peritoneum: 3-0 polyglactin running suture; fascia: 0 polyglactin running suture; subcutaneous layer: 3-0 polyglactin running suture; skin: skin staplers after kidney retrieval surgery, to facilitate re-opening the abdomen the 
following day and 2-0 polypropylene single knot sutures after the transplantation procedure for definitive closure.

21. After applying sterile wound dressing, return the animal to the housing facility and allow to recover following endotracheal extubation. For postoperative analgesia, use buprenorphine $(0.05-0.1 \mathrm{mg} / \mathrm{kg})$ intramuscularly every $8 \mathrm{~h}$ until auto-transplantation.

\section{Back-table and organ preservation}

1. After graft retrieval, immediately cannulate the renal artery using a standard $14 \mathrm{G}$ (orange) peripheral catheter and fix it using a tourniquet prepared from 3-0 polyglactin.

2. Rinse the kidney with cold organ preservation solution.

3. After flushing with $500 \mathrm{~mL}$ of organ preservation solution, remove the arterial cannula, wrap the kidney graft in sterile organ bags and store in organ preservation solution with a target cold ischemic time (CIT) of $24 \mathrm{~h}$ at $4{ }^{\circ} \mathrm{C}$ using a computer-controlled cooling circuit.

NOTE: A brief post-preservation flush is recommended using $500 \mathrm{~mL}$ of $4{ }^{\circ} \mathrm{C}$ normal saline solution.

\section{Contralateral nephrectomy and orthotopic kidney auto-transplantation}

1. During the recipient operation, adapt premedication and initial anesthesia to the restricted renal metabolism and avoid the use of ketamine. Induction is performed with propofol (3-5 mg/kg i.v.), midazolam (0,25 mg/kg i.v.), and atropine $(0.1 \mathrm{mg} / \mathrm{kg}$ i.m. $)$. Thereafter, the preoperative preparation is identical to the procedures described in section 2.

2. Maintain anesthesia with isoflurane (final expiratory 1.45-2.0 Vol.\%) and fentanyl (3 - 7,5 $\mu \mathrm{g} / \mathrm{kg} / \mathrm{h})$ and propofol (2 - $4 \mathrm{mg} / \mathrm{kg} / \mathrm{h})$.
3. Check and continuously monitor ECG, pulse oximetry, rectal temperature and the function of the telemetry transponder.

NOTE: Strict anesthesia and blood pressure control is of crucial importance during the implantation procedure.

4. In rare cases where the arterial blood pressure signal registered over the telemetry transponder is not satisfactory due to the supine position of the animal, place a further arterial catheter into the right femoral artery using percutaneous puncture and the Seldinger technique.

5. Following sterile draping, reopen the median laparotomy and expose the surgical field using the abdominal retractor. The colon and small bowel are placed to the left side of the abdomen to expose the intact right kidney.

6. Similar to the donor procedure, dissect the contra-lateral kidney and its vessels from the surrounding tissue. Dissect the right renal vein and renal artery in the direction of the kidney hilum to ensure sufficient vessel length for anastomosis.

7. 5 min before vascular clamping, inject natrium-heparin intravenously (100 I.U./ kg).

8. Clamp the right renal artery and the right renal vein using vascular clamps. The right kidney is removed. The vessels are checked for integrity before starting the anastomoses.

9. Place the preserved graft kidney into the abdomen and start the venous and arterial anastomoses.

10. From this point onwards, keep the mean arterial pressure over $80-90 \mathrm{~mm} \mathrm{Hg}$ to ensure a good early perfusion of the kidney graft following reperfusion. Achieve this partially by adequate volume management and partially by the administration of norepinephrine $(0.1-1.0 \mu \mathrm{g} / \mathrm{kg} /$ 
min as a continuous infusion using the mean arterial blood pressure and heart rate for monitoring the efficiency).

11. Perform end-to-end anastomosis of the renal vein:

1. After placing two corner stitches using 5-0 polypropylene, suture the back wall in a continuous fashion.

2. Tie the cranial corner stitch and tie it together with the thread used for the back wall.

3. After finishing the back wall, use the cranial corner stitch to suture the front wall in a cranio-caudal direction. Flush the vein with aheparinized saline solution (100 I.U./mL). Tie the caudal corner stitch.

NOTE: In case of a size mismatch between the donor and recipient sides, a small growth-factor can be used to ensure a wide and sufficient anastomosis. There are many possible variations of the porcine renal vein branches. In the case of complex venous anatomy, a modified anastomosis approach is necessary (see Figure 3).

12. Perform the end-to-end anastomosis of the renal artery:

1. Use a 6-0 polypropylene cranial corner stitch to perform the arterial anastomosis. Placing a further caudal, supporting corner stitch which is later removed, is optional.

2. Suture the back wall in a continuous fashion using the parachute technique. After arriving at the caudal corner remove the second corner stitch (if applicable).

3. Suture the front wall with the other end of the doublearmed 6-0 polypropylene suture. Flush the artery with a heparinized saline solution (100 I.U./mL). Tie the two threads at the caudal corner.
13. Record the time needed for performing both anastomoses with a target warm ischemia time of $<40 \mathrm{~min}$.

14. Reperfuse the kidney by opening the venous vascular clamp and subsequently the arterial clamp. Check for significant bleeding.

15. If no significant bleeding from the anastomoses is observed, unwrap the kidney graft and pour warm normal saline solution in the abdomen covering the reperfused graft.

16. Reposition the graft, if needed, to ensure homogeneous reperfusion and avoid congestion.

17. Administer papaverine topically to the outside of the renal artery and the arterial anastomosis (5 $\mathrm{mL}$ undiluted).

18. After reperfusion, infuse $250 \mathrm{~mL}$ of $20 \%$ glucose solution to induce osmotic dieresis followed by the administration of a single dose of $80 \mathrm{mg}$ of furosemide.

NOTE: Following this, initial urine production may be observed.

19. To ensure urinary drainage, pass a 12 French pediatric urine catheter through the abdominal wall of the right flank of the animal, retroperitoneally.

20. Secure the catheter in the ureter using ligatures (2-0 polyglactin) and block the catheter with $2 \mathrm{~mL}$ saline. Further sutures are used to adapt and secure the ureter to the peritoneum of the abdominal wall (2-0 polypropylene). The catheter is also secured to the skin with at least two single knot sutures (2-0 polypropylene).

21. Close the peritoneal layer over the kidney to prevent dislocation of the kidney graft and kinking of the vascular anastomoses (3-0 polyglactin).

22. Close the abdomen in a similar 4-layer fashion as described earlier for the graft retrieval. 
23. Following abdominal closure, maintain normothermia on the OR table.

NOTE: Mean arterial blood pressure should be maintained over $80 \mathrm{~mm} \mathrm{Hg}$ until the animal is awake and is in a prone position.

24. Following abdominal closure, use color Doppler ultrasound to ensure adequate arterial and venous perfusion of the kidney graft (Figure 4). Monitor the animal closely until it is fully awake and can walk and drink spontaneously. The animals are given $1 \mathrm{~L}$ of Ringer solution during the recovery phase.

25. Subsequently, return the animal to its box in the housing facility.

\section{Follow up, sample and data collection}

1. Provide the animals water ad libitum as soon as they can drink spontaneously. Provide solid food from postoperative day 1 .

2. For postoperative analgesia, administer buprenorphine (0.05 - $0.1 \mathrm{mg} / \mathrm{kg}$ ) intramuscularly every $8 \mathrm{~h}$ for $72 \mathrm{~h}$, give pantozol (40mg i.v.) once a day for 72 h. Provide antibiotic treatment (cefuroxime $35 \mathrm{mg} / \mathrm{kg}$ i.v. 2x daily) and thrombosis prophylaxis (500 mg of acetylsalicylic acid from postoperative day 1) during the whole observation period until the end of the experiment.

NOTE: If bleeding complications occur, aspirin is discontinued.

3. Register continuous telemetry data throughout the observation period. Ensure that the animals are visited at least every $8 \mathrm{~h}$ by the veterinary officer and/or by an experienced veterinary technician and their clinical condition is evaluated using a score sheet which is used as a basis to prematurely terminate the experiment if required by the clinical condition of the animal.

NOTE: These so-called humane endpoint criteria are defined as described previously ${ }^{24}$.

4. Perform daily sample collection using the central venous line and the percutaneous urinary catheter. Change urinary bags $(2,000 \mathrm{~mL}) 2 x$ daily.

5. Following sample collection or the administration of fluids or drugs, block the central venous catheter with heparinized saline solution (100 I.U./mL) between every use to avoid occlusion and cover it with a new sterile cap.

6. Following the corresponding observation period of 5 to 7 days, sacrifice the animals in deep anesthesia following relaparotomy, sample collection and explantation of the kidney graft. Sacrifice is performed using a single injection of pentobarbital (50 - $60 \mathrm{mg} / \mathrm{kg}$ i.v.).

NOTE: In compliance with the $3 R$ principle, the remaining organs and tissues of the sacrificed animals may be used for various ex vivo research and educational purposes in in-house institutes.

\section{Representative Results}

Our group has several years of experience with solid organ transplantation models in small- and large animals and utilized the porcine orthotopic kidney auto-transplantation model, obtaining reproducible results in various experimental settings $16,25,26,27$. Depending on the experimental setup, we recommend performing 3 to 5 auto-transplantations as preliminary experiments which ensures a sufficient learning curve of the whole experimental team. In the present setting 5 transplantations were required to train a surgeon, with 8 years of previous experimental- and 5 years of clinical surgical experience in the field of transplantation surgery, in 
performing these experiments. This can differ depending on the previous exposure of the surgeon to these techniques.

Within the frameworks of this protocol, the results of a set of 5 porcine orthotopic kidney auto-transplantation experiments are demonstrated. Transponder implantation was successful in each animal with sufficient telemetry signals throughout the observation period (except one animal with partial transponder dysfunction). Knife-to-skin interval for the transponder implantation was $85 \min \pm 5 \min$ (Table 1). Following graft retrieval, all animals recovered well in the housing facility. Knife-to-skin interval for the retrieval surgery was $135 \mathrm{~min} \pm 32 \mathrm{~min}$ (including approximately $30-45 \mathrm{~min}$ for the insertion, tunneling and securing of the jugular catheter). The left kidney was stored in a cold water-bath with a target cold ischemia time of $24 \mathrm{~h}(24 \mathrm{~h} \pm 30 \mathrm{~min})$. The following day, after anesthesia induction and relaparotomy, the contralateral (right) kidney was removed followed by the orthotopic autotransplantation of the cold stored left kidney graft as described earlier. Knife-to-skin interval for the auto-transplantation surgery was $168 \min \pm 27$ min (including the explantation of the right kidney). Warm ischemia time was $34 \min \pm 7$ min. Each implanted kidney graft had a minimal but direct urine production following reperfusion. Following abdominal closure, color Doppler ultrasound showed satisfactory arterial and venous perfusion of the kidney in all cases (Figure 4). All animals recovered from the anesthesia and no significant complications were observed throughout the observation period. Daily blood and urine samples were collected. All pigs were in good clinical condition during the follow-up and were sacrificed after 5 days. Serum creatinine and potassium values peaked on POD3-4. The blood $\mathrm{pH}$ has remained within normal ranges (Figure 5). Urine output recovered to normal values over the first four postoperative days. White blood cell count was slightly increased at the end of the follow-up period (Figure 5). Body temperature, measured by continuous telemetry monitoring, showed slight fluctuations over the postoperative period.

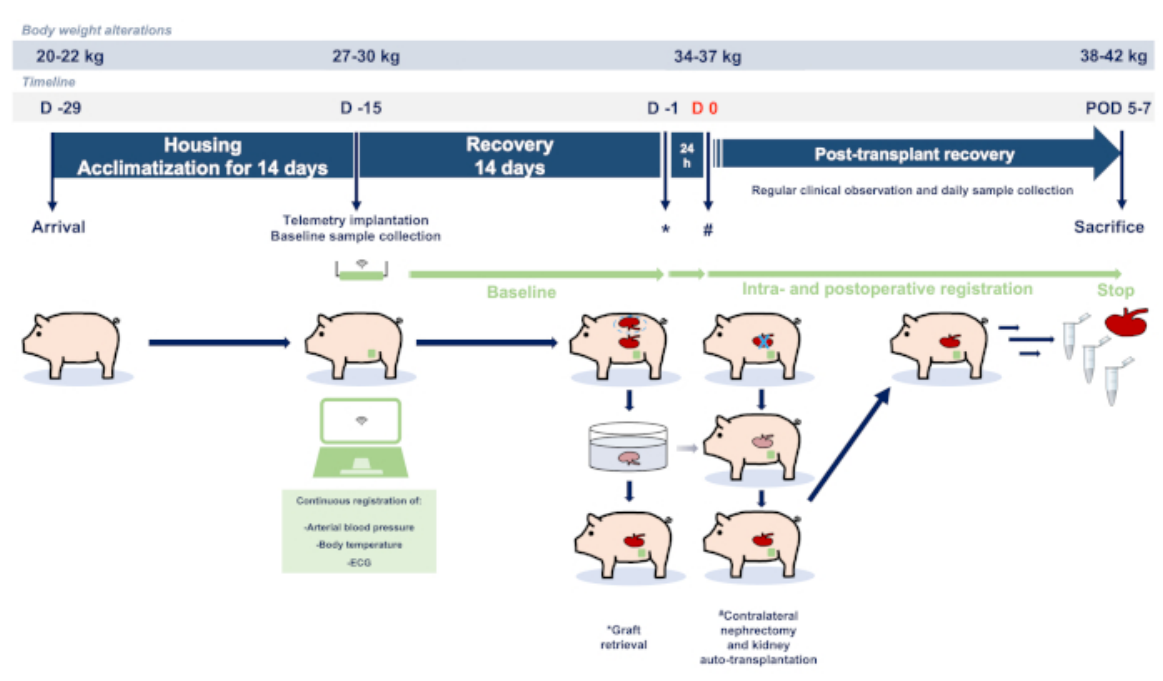

Figure 1: Study flowchart and protocol. Abbreviations used: POD-postoperative day; ECG-electrocardiography. Please click here to view a larger version of this figure. 

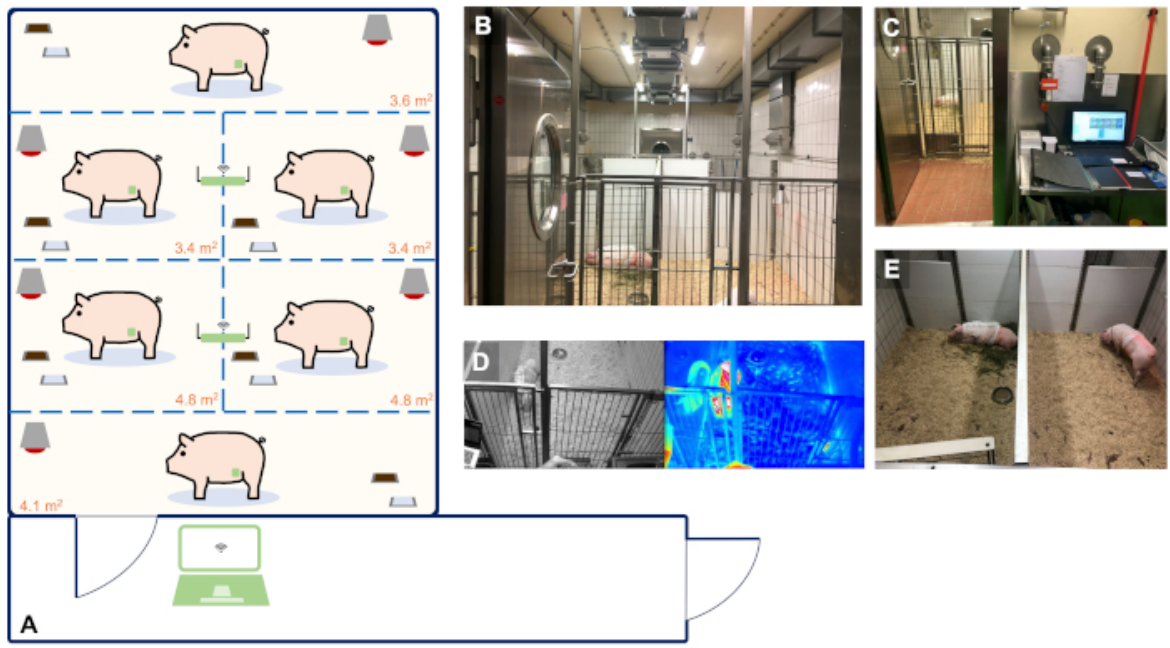

Figure 2: Animal housing facility with real-time and continuous telemetry monitoring of up to 6 animals. (A)

Schematic blueprint of our facility suitable for the housing and telemetry monitoring of up to 6 animals. The size of the single holding boxes was determined based on the guidelines of the EU Directive 2010/63 and ETS 123 Appendix A. Panels A-E show representative images of the organization of our facility. (B) Animal room for the housing of 6 animals. (C) Observation room with a PC used for the continuous registration of telemetry data. (D) Real-time video and thermal footage of the animals. (E) Individual holding ensuring acoustic and olfactory contact of the animals with their companions to avoid social isolation. Please click here to view a larger version of this figure. 

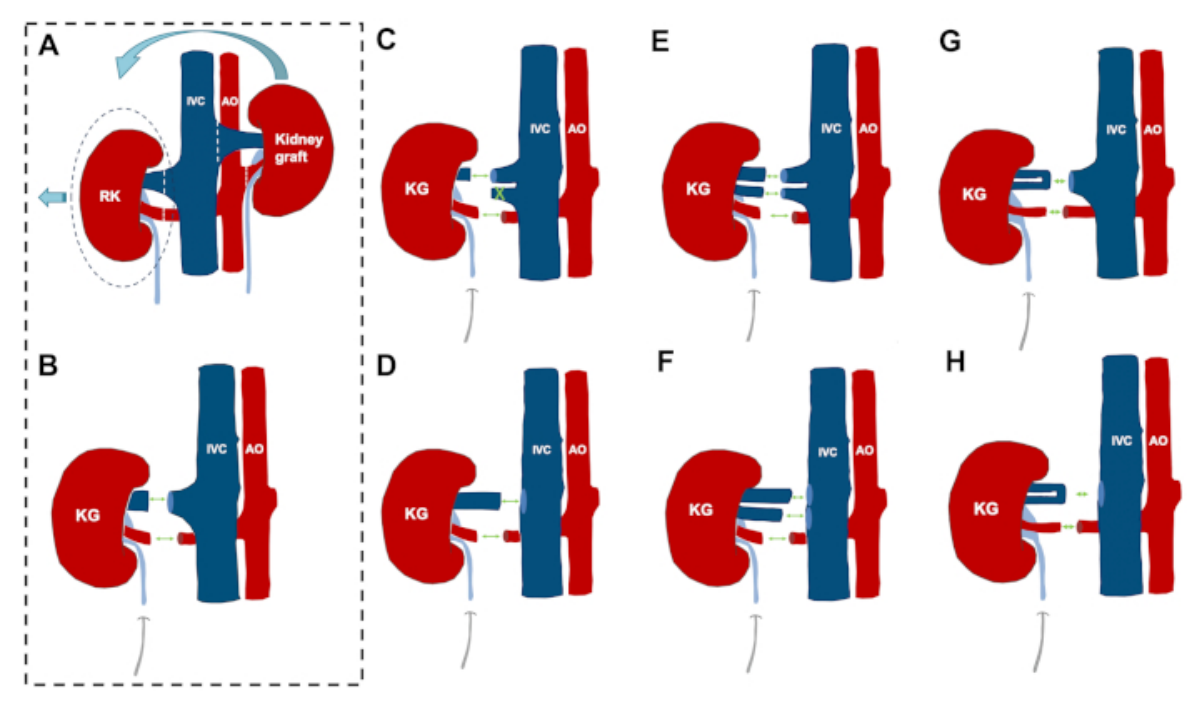

Figure 3: Orthotopic kidney auto-transplantation and anatomical variations and reconstruction possibilities. (A,B)

The steps of the orthotopic kidney auto-transplantation model in case of a "standard" vascular anatomy. (C) Variation 1 : while one larger vein comes with the donor kidney, there are two veins on the recipient side. Management: the smaller vein is closed by a ligature and the anastomosis is performed end to end between the renal veins. (D) Variation 2: while one larger vein comes with the donor kidney, there is no suitable recipient vessel on the contralateral side (e.g., size mismatch). Management: end to side anastomosis of the renal vein to the inferior vena cava. (E) Variation 3: two similar-sized veins on both sides. Management: reconstruction by two venous anastomoses. (F) Variation 4: while two similar-sized veins come with the donor kidney, there is no suitable recipient vessel on the contralateral side. Management: end to side anastomosis of the renal vein to the inferior vena cava in the case of two renal veins. (G) Variation 5: a donor kidney comes with a vein showing an early bifurcation, while there is one large vein on the contralateral side. Management: end to end anastomosis of the short common channel of the donor renal vein with one large vein on the recipient side. $(\mathbf{H})$ Variation 6: while the donor kidney comes with a single renal vein with an early bifurcation, there is no suitable recipient vessel on the contralateral side. Management: end to side anastomosis of the short common channel of the donor renal vein to the inferior vena cava. This figure depicts a handful of the more frequent variations and is not statistically comprehensive in terms of all variations possible in German landrace pigs. Abbreviations used: KG-kidney graft; RK-right kidney; IVC-inferior vena cava; AO-aorta Please click here to view a larger version of this figure. 


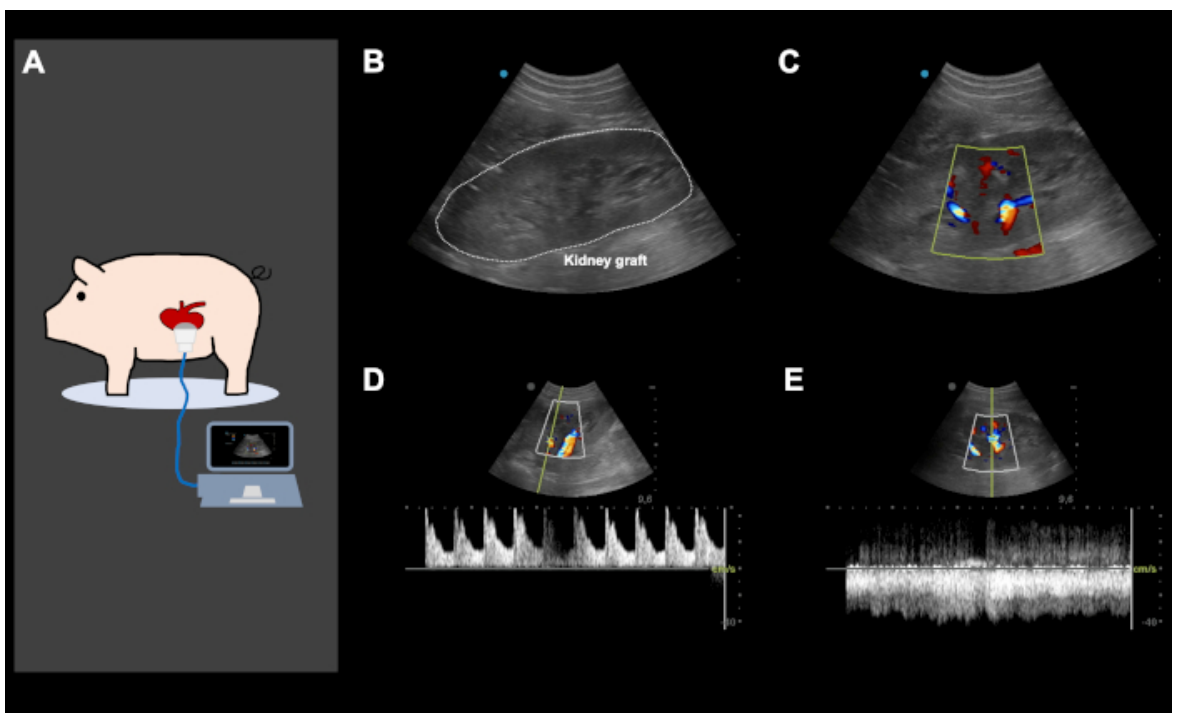

Figure 4: Representative color Doppler ultrasound images, directly after orthotopic kidney auto-transplantation and abdominal closure. (A) Color Doppler ultrasound is performed directly following the implantation of the kidney and abdominal closure, to ensure good arterial and venous perfusion of the kidney graft and to screen for potential iatrogenic vascular kinking. Ultrasound was also used daily and on-demand, based on the clinical performance of the animal to screen for various problems. (B-E) Representative ultrasound images of a kidney graft following implantation. The image of the kidney graft with and without color Doppler (B,C) shows an excellent arterial (D) and venous perfusion (E). This figure show representative images from the same animal. Please click here to view a larger version of this figure. 
A

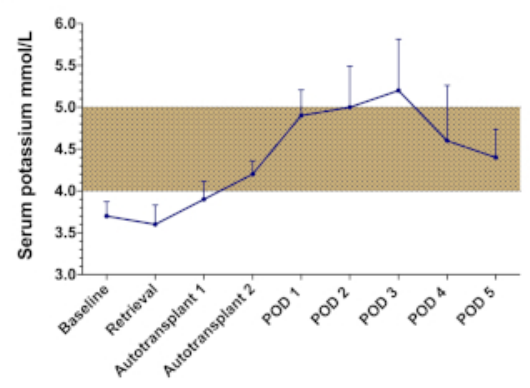

C

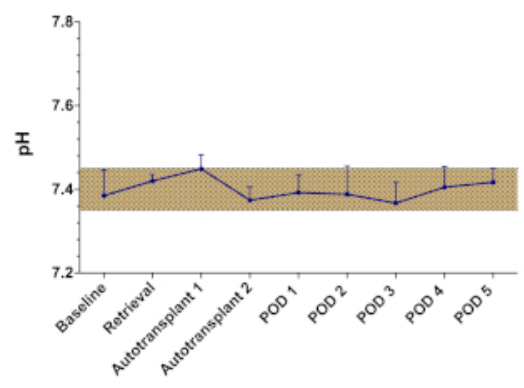

E

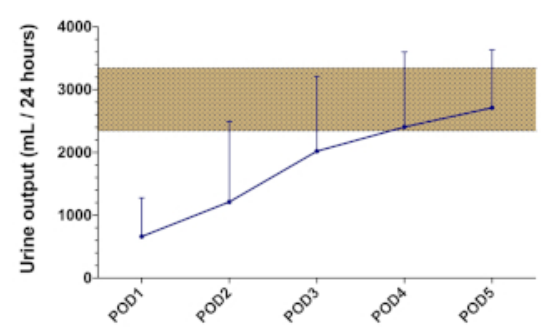

B

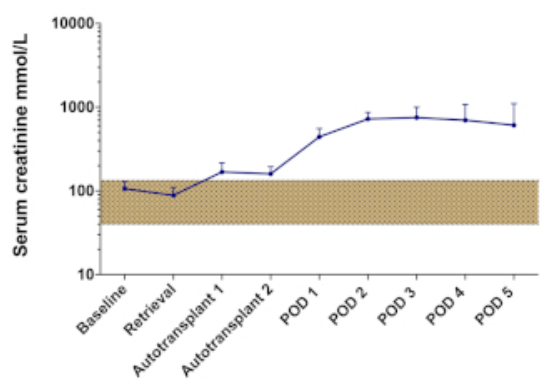

D

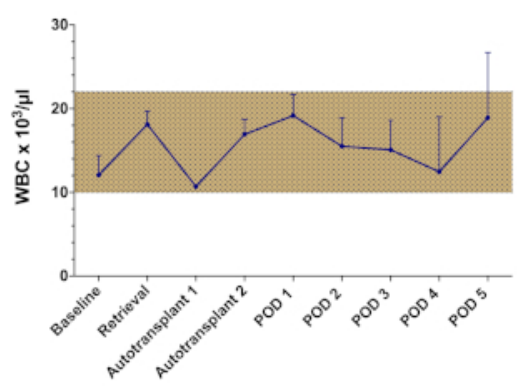

$\mathbf{F}$

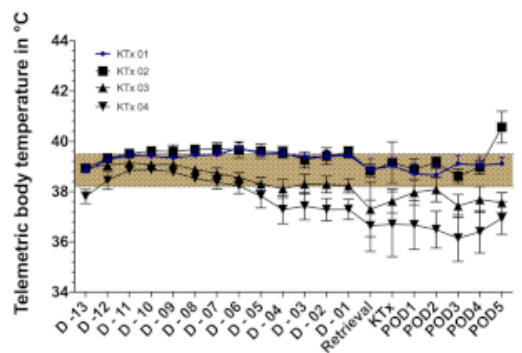

Figure 5: Representative laboratory findings and telemetry data of the orthotopic kidney auto-transplantation model with a cold ischemia time of $\mathbf{2 4} \mathbf{h}$. (A) Serum potassium values (B) Serum creatinine values (C) pH (D) White blood cell count (WBC) (E) Urine output. (F) Mean body temperature registered by telemetric monitoring throughout the observation period in four consecutive kidney transplantation (no data presented from the $5^{\text {th }}$ animal due to partial transponder dysfunction). Abbreviations used: POD-postoperative day. Please click here to view a larger version of this figure. 


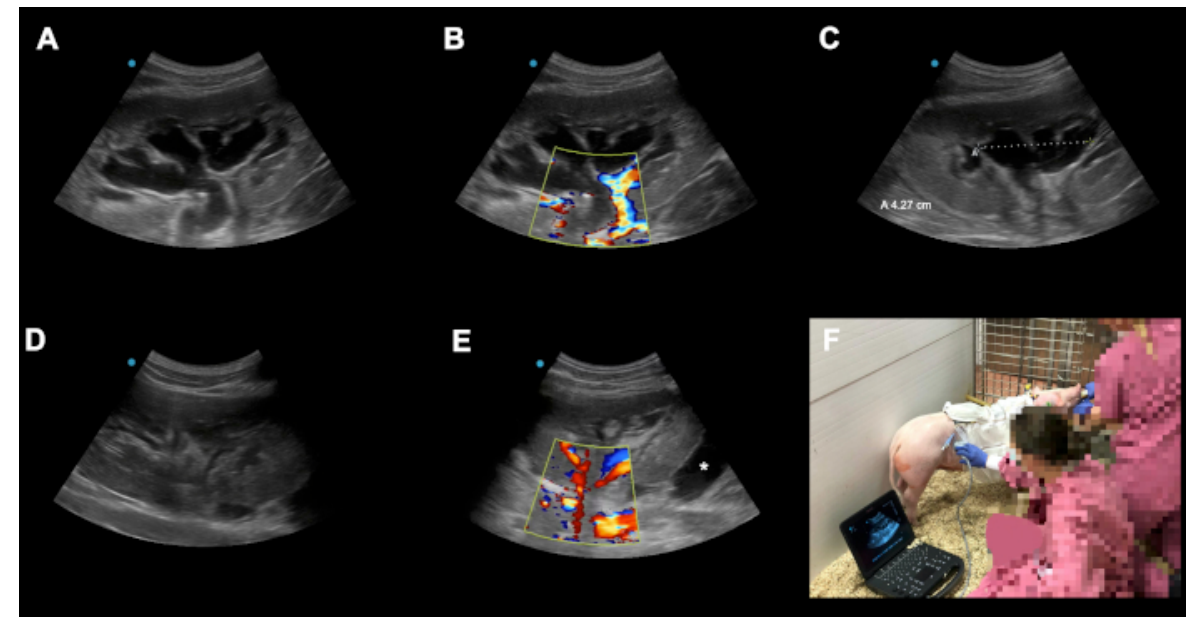

Figure 6: Examples of possible peri-operative complications and pitfalls. (A-C) Postoperative congestion of the transplanted kidney graft on POD3 following orthotopic kindey auto-transplantation. (D) The reason for the congestion was identified as catheter kinking due to an overtightened suture on the level of the skin. After readjusting the suture the congestion resolved almost completely in $24 \mathrm{~h}$. (E) Here an other kidney graft on POD2 following orthotopic kidney auto-transplantation is shown. Asterix $\left({ }^{*}\right)$ shows a fluid collection around the underpole of the graft (bloody collection vs. lymphocele). Because of our technique with closure of the peritoneum over the kidney these collections are usually self limiting due to the advantageous effects of local compression. Animals should be monitored closely in terms of the local finding, signs of bleeding or infection. (F) Qualified color Doppler ultrasound performed daily (and on demand) in the housing facility has, besides its academic utilization (e.g., documentation, registration of arterial resistence indices), a crucial diagnostic role in recognizing potential complications in the early subclinical phase. Please click here to view a larger version of this figure. 


\begin{tabular}{|c|c|c|c|c|c|c|c|c|}
\hline \multirow{2}{*}{$\begin{array}{l}\text { Experimental } \\
\text { task/step }\end{array}$} & \multirow[t]{2}{*}{ Days } & \multirow[t]{2}{*}{ Time (min) } & \multirow[t]{2}{*}{ Surgeon } & \multirow{2}{*}{$\begin{array}{c}\text { Veterinary } \\
\text { officer }\end{array}$} & \multirow{2}{*}{$\begin{array}{l}\text { Veterinary } \\
\text { technician }\end{array}$} & \multirow{2}{*}{$\begin{array}{l}\text { Laboratory } \\
\text { technician }\end{array}$} & \multirow{2}{*}{$\begin{array}{l}\text { Doctoral } \\
\text { student }\end{array}$} & \multirow{2}{*}{$\frac{\text { Total }}{\mathrm{Nr}}$} \\
\hline & & & & & & & & \\
\hline $\begin{array}{c}\text { Preopreative } \\
\text { care }\end{array}$ & $\mathrm{D}-29$ to $\mathrm{D}-15$ & n.a. & & 1 & 1 & & 1 & 3 \\
\hline $\begin{array}{l}\text { Telemetry } \\
\text { implantation } \\
\text { surgery }\end{array}$ & D-15 & $85 \pm 5$ & 1 & 1 & 1 & 1 & 1 & 5 \\
\hline $\begin{array}{l}\text { Postoperative } \\
\text { care following } \\
\text { telemetry } \\
\text { implantation }\end{array}$ & D-15 to D-1 & n.a. & & 1 & 1 & & 1 & 3 \\
\hline $\begin{array}{c}\text { Graft retrieval } \\
\text { surgery }\end{array}$ & D-1 & $135 \pm 32$ & 1 & 2 & 1 & 2 & 2 & 8 \\
\hline $\begin{array}{c}\text { Kidney auto- } \\
\text { transplantation } \\
\text { surgery }\end{array}$ & D 0 & $168 \pm 27$ & 1 & 2 & 1 & 2 & 2 & 8 \\
\hline $\begin{array}{l}\text { Postoperative } \\
\text { care following } \\
\text { kidney auto- } \\
\text { transplantation }\end{array}$ & D 0 to D5 & n.a. & & 2 & 1 & & 2 & 5 \\
\hline Sacrifice & D 5 & n.a. & & 2 & & 1 & 1 & 4 \\
\hline
\end{tabular}

Table 1. Description of the required human resources and time-schedules for performing various experimental steps of the porcine kidney auto-transplantation model.

\section{Discussion}

The porcine model of KT allows the investigation of novel therapeutic approaches and medical devices in a clinically relevant large animal setting $15,17,21$. The anatomical, pathophysiological and surgical-technical similarities between the porcine and human setting can facilitate the clinical interpretation of data and the rapid translation of the findings and techniques into clinical testing $15,16,17,18,19,21$.

The model of orthotopic kidney auto-transplantation does not only comply with the $3 R$ principle by reducing the numbers of required animals compared to allo-transplantation, e.g. no separate donor animal is required, but also provides a unique opportunity to investigate the effects of IRI and preservation 
injury without the confounding effects of the immunological response and immunosuppressive drugs ${ }^{17,21}$.

Slight modifications of the protocol allow modeling a broad spectrum of clinical situations. To mimic KT using donation after circulatory death (DCD) kidneys, vascular structures are clamped for 30 to $60 \mathrm{~min}$ in situ before kidney retrieval, while prolonged cold ischemia times ( 24 hours and longer) can be applied to model extensive preservation injury $16,17,28,29$.

Although, the porcine KT model is surgically less challenging than solid organ transplantation models in small animals (e.g. rats and mice $)^{26}$, there are multiple technical aspects and pitfalls which have to be kept in mind to improve outcomes and avoid specific complications ${ }^{17}$.

Failing to avoid the large lymphatic vessels around the inferior vena cava and the aorta during graft retrieval or implantation due to technical mistake or anatomical variations, can lead to a high output lymphatic fistula and post-operative abdominal fluid collection, infection, and potentially technical failure. Lymphatic vessels should be completely avoided during surgery or closed with 5-0 or 6-0 polypropylene sutures. It is wise to also avoid the use of bipolar or any other coagulation device in case of lymphatic leaks. It usually leads to worsening of the situation. In case of a low output lymphatic leakage, our team has a good experience with the application of fibrin-based collagen patches (e.g., Tachosil) ${ }^{30}$, however, their high cost limits their application in this setting.

In the present protocol we demonstrate a transperitoneal approach for kidney retrieval and auto-transplantation. This is a major technical difference compared to the clinical situation, where kidney grafts are usually implanted into the iliac fossa using an extraperitoneal approach. Although, most groups use a transperitoneal and an orthotopic approach in the porcine model, heterotopic transplantation to the iliac fossa is also possible in pigs $^{31}$. However, due to the relatively low diameter of the external iliac artery in $30-40 \mathrm{~kg}$ pigs and its tendency to vasospasm makes it sometimes difficult to perform the end-to-side anastomosis of the renal artery to the external iliac artery ${ }^{31}$. Concerning the fact that we retrieve the left kidney via a transperitoneal approach to perform a subsequent auto-transplantation, it is more feasible to perform the implantation by reopening the same incision and using a straigtforward orthotopic approach, especially that per-protocol it is also required to remove the native right kidney to ensure that the animal will recover with only one predamaged kindey. The comprehensive description of all possible technical variations of the model is beyond the scope of this protocol and has been summarized by others in comprehensive review articles ${ }^{31}$.

Dislocation of the transplanted kidney graft and consequential kinking of the vascular anastomoses is a major source of failure in the porcine KT model, rapidly leading to vascular occlusion and complete failure of the experiment, due to a surgical complication. To avoid this, following autotransplantation we close the peritoneal layer over the kidney with a running suture using 3-0 polyglactin. Furthermore, color Doppler ultrasound is performed directly following the implantation of the kidney and abdominal closure, to ensure good arterial and venous perfusion of the kidney graft. Ultrasound is also used daily and on-demand, based on the clinical performance of the animal, to screen for kidney perfusion, post-renal problems (e.g. obstruction or kinking of the urinary catheter), and fluid collection due to lymphatic fistula, bleeding or infection (Figure 4 and Figure 6).

As 24 hours of cold ischemia often leads to functional impairment and delayed graft function, the animals may 
require on-demand medical therapy if it is considered necessary by the veterinary officer. This may include infusion therapy using 5\% glucose and/or Ringer solution administered via the central venous line, furosemide bolus injections (in case of oliguria/anuria depending on the clinical state and laboratory results, $60-80 \mathrm{mg}$ bolus injections up to $200 \mathrm{mg} / \mathrm{day}$ ), and the oral administration of Sodium Polystyrene Sulfonate (Resonium A) in case of severe hyperkalemia $^{32}$. To avoid experimental bias, the veterinary officer responsible for the post-transplant veterinary care of the animals must be blinded for the applied treatment and grouping.

Although, the anatomy of the renal artery is rather straightforward in German landrace pigs with usually one artery to reconstruct, there is a wide spectrum of anatomical variations of the renal vein branches which require certain surgical creativity during the venous reconstruction. Frequently two (or more) renal vein branches join on different levels between the kidney hilum and the inferior vena cava. The most frequently observed variations and the possible reconstruction options ${ }^{17}$ are shown in Figure 3.

Following the first surgical intervention (day -15 , telemetry implantation), all animals receive a porcine jacket which they wear throughout the whole period of the experiments. This provides excellent protection against accidental injuries and dislocation of the implanted catheters and provides room for the storage of the urine collection bags. The use of these jackets is also a feasible solution to eliminate the need for metabolic cages for the assessment of creatinine clearance as a refinement method according to the $3 \mathrm{R}$ Principle.

Our housing facility integrates the use of telemetry and videobased peri-operative monitoring. Although, these methods cannot replace the regular visits by the veterinary officer and technicians, they facilitate rapid interventions and improve severity assessment to further refine our experimental settings for the future. There is a wide spectrum of indications for the use of an implantable telemetry device in large animal models ${ }^{33}$. Although, close monitoring of clinical paramters following major surgery such as ECG, blood pressure, temperature is considered to be standard in the human clinical setting of a surgical intensive- and intermediate care unit, in experimental surgery monitoring is mostly discontinued when the animal is waking up from anaesthesia $33,34,35$. Therefore, telemetry provides a feasible way for the continuous monitoring of these animals. We believe that all these data contribute to the early detection of possible postoperative complication accurately and timely (e.g., haemorrhagic shock, or sepsis detected by increasing temperature, hypotonia and tachycardia). This may facilitate timely intervention (e.g., introduction of therapeutic antibiotic therapy, fluid substitution, discontinuation of anticoagulation, or sacrifice of the animal to avoid suffering). Besides these "real-time" monitoring aspect, our group is currently focusing on the severity assessment and refinement of animal experiments $36,37,38$. Retrospective analysis of a large amount of collected telemetry data in these experiments may allow us to better stratify the severity of these kind of surgical interventions and optimize perioperative care (e.g., analgesia) in laboratory animals.

In terms of implantable telemetry, a period of at least 12 days after implantation of the measurement system is recommended to ensure stable and optimal measurement data (based on personal communication). After discussing this issue with various manufacturers providing telemetry solutions for large animals as well as with other research groups using these systems in various experimental settings, we decided to integrate a 14 day period between telemetry 
implantation and kidney transplantation. During the earlier days, deviations may still occur due to the movement of the animal as the scarring and healing processes are still uncomplete.

Despite its advantages, the above-described model has certain limitations. The complexity and required resources and infrastructure are the most important limitations of the model. The time-consuming experimental protocol, complex techniques, and intense peri-operative follow up necessitate the availability of a significant housing and OR capacity and require the involvement of a larger team, including doctoral fellows, surgeons, veterinary officers, and technicians (Table 1). Therefore, based on our empirical observations, it is usually unfeasible to perform more than two procedures a day. A further disadvantage of the porcine model compared to small animal models is the limited possibility of mechanistic and molecular-biological investigations. In the present protocol only 5-days of follow up was reported. This was suitable to demonstrate the most important experimental characteristics of the model, however, this relatively short follow up may not be sufficient to answer certain specific research question (e.g. long-term recovery of function vs. acute damage). Therefore, a project related extension of the follow up might be necessary. This manuscript describes our current "best-practice" in the experimental setting of porcine orthotopic kidney auto-transplantation. While certain steps are mandatory to successfully establish this model, minor aspects (e.g., the intraoperative use of a bladder catheter, arterial catheter placement to the femoral vs. carotid artery) are facultative and may be avoided/altered at the investigators discretion. Description and justification of each and every methodical aspect would be beyond the scope of the present protocol and has been discussed elsewhere ${ }^{31}$. Finally, it is also difficult to replicate the exact clinical situation of ECD KT in the porcine model where elderly donors, allografts with acute kidney injury and donors with multiple co-morbidities and chronic diseases such as hypertension, diabetes mellitus or arteriosclerosis represent a major part of the marginal donor pool 8,9 .

Notwithstanding the above-mentioned limitations as well as technical and logistical challenges, this well-established and reproducible large animal model of $\mathrm{KT}$ provides a unique opportunity to investigate novel therapies and techniques to improve organ preservation and clinical outcomes and represents an excellent platform for younger surgeons to master organ transplantation techniques in a large animal model.

\section{Disclosures}

The authors have no conflict of interest to disclose.

\section{Acknowledgments}

The authors would like to express their gratitude to Pascal Paschenda, Mareike Schulz, Britta Bungardt, Anna Kümmecke for their skillful technical assistance.

The authors declare funding in part from the START program of the Faculty of Medicine, RWTH Aachen University (\#23/19 to Z.C.), from the B.Braun Foundation, Melsungen, Germany (BBST-S-17-00240 to Z.C.), the German Research Foundation (Deutsche Forschungsgemeinschaft - DFG; FOR-2591, TO 542/5-1, TO 542/6-1; 2016 to R.T. and SFB/ TRR57, SFB/TRR219, BO3755/3-1, BO3755/6-1 to P.B.) and the German Ministry of Education and Research (BMBF: STOP-FSGS-01GM1901A to P.B.), without the involvement of the funders in study design, data collection, data analysis, manuscript preparation or decision to publish. 


\section{References}

1. Watts, G. Joseph Murray: innovative surgeon and pioneer of transplantation. Lancet. 377 (9770), 987 (2011).

2. Merion, R. M. et al. Deceased-donor characteristics and the survival benefit of kidney transplantation. Journal of the American Medical Association. 294 (21), 2726-2733 (2005).

3. Jochmans, I., O'Callaghan, J. M., Pirenne, J., Ploeg, R. J. Hypothermic machine perfusion of kidneys retrieved from standard and high-risk donors. Transplant International. 28 (6), 665-676 (2015).

4. Czigany, Z. et al. Machine perfusion for liver transplantation in the era of marginal organs-New kids on the block. Liver International. 39 (2) 228-249 (2018).

5. Fabrizii, V. et al. Patient and graft survival in older kidney transplant recipients: does age matter? Journal of the American Soceity of Nephrology. 15 (4), 1052-1060 (2004).

6. Jochmans, I., Nicholson, M. L., Hosgood, S. A. Kidney perfusion: some like it hot others prefer to keep it cool. Current Opinion in Organ Transplantation. 22 (3), 260-266 (2017).

7. (DSO), D. S. O. DSO Jahresbericht 2018. <https://www.dso.de/SiteCollectionDocuments/ DSO_Jahresbericht_2018.pdf> (2018).

8. Meister, F. A. et al. Hypothermic Oxygenated Machine Perfusion of Extended Criteria Kidney Allografts from Brain Dead Donors: Protocol for a Prospective Pilot Study. JMIR Research Protocols. 8 (10), e14622 (2019).

9. Meister, F. A. et al. Hypothermic oxygenated machine perfusion-Preliminary experience with end-ischemic reconditioning of marginal kidney allografts. Clinical Transplantation. 33 (10), e13673 (2019).

10. Siedlecki, A., Irish, W., Brennan, D. C. Delayed graft function in the kidney transplant. American Journal of Transplantation. 11 (11), 2279-2296 (2011).

11. Plenter, R., Jain, S., Ruller, C. M., Nydam, T. L., Jani, A. H. Murine Kidney Transplant Technique. Journal of Visualized Experiments. (105), e52848 (2015).

12. Fabry, G. et al. Cold Preflush of Porcine Kidney Grafts Prior to Normothermic Machine Perfusion Aggravates Ischemia Reperfusion Injury. Scientific Reports. 9 (1), 13897 (2019).

13. Kalenski, J. et al. Improved preservation of warm ischemia-damaged porcine kidneys after cold storage in Ecosol, a novel preservation solution. Annals of Transplantation. 20 233-242 (2015).

14. Kalenski, J. et al. Comparison of Aerobic Preservation by Venous Systemic Oxygen Persufflation or Oxygenated Machine Perfusion of Warm-Ischemia-Damaged Porcine Kidneys. European Surgical Research. 57 (1-2), 10-21 (2016).

15. Kaths, J. M. et al. Normothermic Ex Vivo Kidney Perfusion for the Preservation of Kidney Grafts prior to Transplantation. Journal of Visualized Experiments. (101), e52909 (2015).

16. Schreinemachers, M. C. et al. Improved preservation and microcirculation with POLYSOL after transplantation in a porcine kidney autotransplantation model. Nephrology Dialysis Transplantation. 24 (3), 816-824 (2009).

17. Kaths, J. M. et al. Heterotopic Renal Autotransplantation in a Porcine Model: A Step-by-Step Protocol. Journal of Visualized Experiments. (108), 53765 (2016). 
18. De Deken, J. et al. Postconditioning effects of argon or xenon on early graft function in a porcine model of kidney autotransplantation. British Journal of Surgery. 105 (8), 1051-1060 (2018).

19. Faure, A. et al. An experimental porcine model of heterotopic renal autotransplantation. Transplantation Proceedings. 45 (2), 672-676 (2013).

20. Golriz, M. et al. Do we need animal hands-on courses for transplantation surgery? Clinical Transplantation. 27 Suppl 25, 6-15 (2013).

21. Gallinat, A. et al. Transplantation of Cold Stored Porcine Kidneys After Controlled Oxygenated Rewarming. Artificial Organs. 42 (6), 647-654 (2018).

22. Russell, W. M. S., Burch, R. L. The Principles of Humane Experimental Technique. Methuen, (1959).

23. Kilkenny, C., Browne, W. J., Cuthill, I. C., Emerson, M., Altman, D. G. Improving bioscience research reporting: the ARRIVE guidelines for reporting animal research. PLoS Biology. 8 (6), e1000412 (2010).

24. Hagemeister, K. et al. Severity assessment in pigs after partial liver resection: evaluation of a score sheet. Laboratory Animals. 54 (3) (2019).

25. Doorschodt, B. M. et al. Hypothermic machine perfusion of kidney grafts: which pressure is preferred? Annals of Biomedical Engineering. 39 (3), 1051-1059 (2011).

26. Czigany, Z. et al. Improving Research Practice in Rat Orthotopic and Partial Orthotopic Liver Transplantation: A Review, Recommendation, and Publication Guide. European Surgical Research. 55 (1-2), 119-138 (2015).

27. Nagai, K., Yagi, S., Uemoto, S., Tolba, R. H. Surgical procedures for a rat model of partial orthotopic liver transplantation with hepatic arterial reconstruction. Journal of Visualized Experiments. (73), e4376, (2013).

28. Doorschodt, B. M. et al. Evaluation of a novel system for hypothermic oxygenated pulsatile perfusion preservation. The Internation Journal of Artificial Organs. 32 (10), 728-738 (2009).

29. Kaths, J. M. et al. Continuous Normothermic Ex Vivo Kidney Perfusion Is Superior to Brief Normothermic Perfusion Following Static Cold Storage in Donation After Circulatory Death Pig Kidney Transplantation. American Journal of Transplantation. 17 (4), 957-969 (2017).

30. Tammaro, V. et al. Prevention of fluid effusion in kidney transplantation with the use of hemostatic biomaterials. Transplantation Proceedings. 46 (7), 2203-2206 (2014).

31. Golriz, M. et al. Pig kidney transplantation: an up-todate guideline. European Surgical Research. 49 (3-4), 121-129 (2012).

32. Higgins, R. et al. Hyponatraemia and hyperkalaemia are more frequent in renal transplant recipients treated with tacrolimus than with cyclosporin. Further evidence for differences between cyclosporin and tacrolimus nephrotoxicities. Nephrology Dialysis and Transplantation. 19 (2), 444-450 (2004).

33. Markert, M. et al. A new telemetry-based system for assessing cardiovascular function in group-housed large animals. Taking the $3 R$ s to a new level with the evaluation of remote measurement via cloud data transmission. Journal of Pharmacological and Toxicological Methods. 93, 90-97 (2018).

34. Längin, M. et al. Perioperative Telemetric Monitoring in Pig-to-Baboon Heterotopic Thoracic Cardiac Xenotransplantation. Annals of Transplantation. 23, 491-499 (2018). 
35. Willens, S., Cox, D. M., Braue, E. H., Myers, T. M., Wegner, M. D. Novel technique for retroperitoneal implantation of telemetry transmitters for physiologic monitoring in Göttingen minipigs (Sus scrofa domesticus). Comparative Medicine. 64 (6), 464-470 (2014).

36. van Dijk, R. M. et al. Design of composite measure schemes for comparative severity assessment in animalbased neuroscience research: A case study focussed on rat epilepsy models. PLoS One. 15 (5), e0230141 (2020).

37. Zieglowski, L. et al. Severity assessment using three common behavioral or locomotor tests after laparotomy in rats: a pilot study. Laboratory Animals. 10.1177/002367722091168023677220911680, (2020).

38. Bleich, A., Bankstahl, M., Jirkof, P., Prins, J. B., Tolba, R. H. Severity Assessment in animal based research. Laboratory Animals. 54 (1), 16 (2020). 